International Journal of Maternal and Child Health and AIDS (2020), Volume 9, Issue I, I 2 I- I 27

\begin{tabular}{ll}
\hline & INTERNATIONAL JOURNAL of \\
& MATERNAL and CHILD HEALTH and AIDS \\
& ISSN 216I-864X (Online) \\
& ISSN 216I-8674 (Print) \\
IJMA Available online at www.mchandaids.org & DOI: 10.21 I 06 /ijma.296 \\
\hline
\end{tabular}

COMMENTARY

\title{
Artificial Intelligence in Global Health -A Framework and Strategy for Adoption and Sustainability
}

Trevor D. Hadley, BSA; ; Rowland W. Pettit, BS; ${ }^{*}$ Tahir Malik, BS; ${ }^{*}$ Amelia A. Khoei, BS;'

Hamisu M. Salihu, MD, PhD ${ }^{2 \bowtie}$

'Baylor College of Medicine, Houston, TX 77098, USA; ${ }^{2}$ Center of Excellence in Health Equity, Training and Research Baylor College of Medicine,

Houston, TX 77098, USA

*These individuals contributed equally as co-first authors.

Corresponding author email: hamisu.salihu@bcm.edu

ABSTRACT

Artificial Intelligence (AI) applications in medicine have grown considerably in recent years. $\mathrm{Al}$ in the forms of Machine Learning, Natural Language Processing, Expert Systems, Planning and Logistics methods, and Image Processing networks provide great analytical aptitude. While Al methods were first conceptualized for radiology, investigations today are established across all medical specialties. The necessity for proper infrastructure, skilled labor, and access to large, well-organized data sets has kept the majority of medical Al applications in higher-income countries. However, critical technological improvements, such as cloud computing and the near-ubiquity of smartphones, have paved the way for use of medical Al applications in resource-poor areas. Global health initiatives (GHI) have already begun to explore ways to leverage medical Al technologies to detect and mitigate public health inequities. For example, Al tools can help optimize vaccine delivery and community healthcare worker routes, thus enabling limited resources to have a maximal impact. Other promising Al tools have demonstrated an ability to: predict burn healing time from smartphone photos; track regions of socioeconomic disparity combined with environmental trends to predict communicable disease outbreaks; and accurately predict pregnancy complications such as birth asphyxia in low resource settings with limited patient clinical data. In this commentary, we discuss the current state of Al-driven GHI and explore relevant lessons from past technology-centered GHI. Additionally, we propose a conceptual framework to guide the development of sustainable strategies for Al-driven $\mathrm{GHI}$, and we outline areas for future research.

Keywords: Artificial Intelligence • Al Framework • Global Health • Implementation • Sustainability - Al Strategy

Copyright @ 2020 Hadley et al. Published by Global Health and Education Projects, Inc. This is an open-access article distributed under the terms of the Creative Commons Attribution License CC BY 4.0. 


\section{Introduction}

Advancements in medical technology have long inspired global health pioneers, who envision ways that new technologies might bridge deficits in skilled labor, infrastructure, and access to resources in developing nations. In the current era, there have been rapid advancements in medically applied Artificial Intelligence (AI), a versatile, complex, and powerful set of tools capable of performing specific tasks which normally require human intelligence. The applications of Al technologies in medicine have grown considerably and are now developed in nearly every medical specialty. ${ }^{1-5}$

However, due to the complexity of medical Al systems, and their reliance on access to unique resources, these applications are applied almost exclusively within the developed world. Despite limitations, there is growing interest in leveraging $\mathrm{Al}$ to address healthcare issues facing the developing world. Proof of concept Al-based Global Health Initiatives (GHI) exists. I gap in knowledge, however, remains regarding how to develop an effective and sustainable approach to Al-based GHI. In this paper, we provide context to the current state of Al-based $\mathrm{GHI}$, exploring pertinent lessons from past technology-centered global health initiatives. We propose a conceptual model to guide the development of sustainable Al models applied in a global health context and provide clear directions for future research endeavors in order to expand upon this framework.

\section{Al and Global Health}

A modest number of Al-based global health initiatives have arisen within the last 10 years. ${ }^{6}$ Several implementation models have been developed for resource-poor settings. ${ }^{7-21} \mathrm{Al}$ model concepts are applicable in: (I) creating intelligent Electronic Health Records (EHR), (2) performing bio-surveillance, (3) diagnosing disease, (4) assisting in clinical decision making, and (5) optimizing planning and scheduling processes.

Design requirements for the creation of large database technologies in low-resource settings have previously been established. ${ }^{22}$ Adapted for Al initiatives, we pose design requirements to include: (I) a model-access to clean, large, and inclusive datasets for model generation; (2) personnel—skilled programmers and data scientists; (3) processesan accurate, continued stream of information flow, and availability of knowledgeable physician/subject matter experts to assess end goal achievement; and (4) infrastructure-a dependable network on which models will run, including access to reliable power and network connectivity. ${ }^{22}$ Achievement of these requirements is the first step toward the realization of a fully formed Al-based GHI. However, to ensure sustainable model deployment, attention must be paid toward durable access to essential resources and sustained capacity for model maintenance. Al is still at baseline a technology that comes with a "principled set of considerations" that will be important for its utilization, standardization, and sustainable deployment in a given $\mathrm{GHI}$.

\section{Lessons from Global Health}

The literature on technology based GHI provides a number of conceptual frameworks to guide effective and sustainable programs. ${ }^{22} \mathrm{~A}$ prominent approach is to break a program down into 'vertical' and 'horizontal' components. To elaborate, a predominately vertical program is one that relies heavily on outsourced labor, resources, and expertise (from developed nations) with minimal integration into local infrastructure..$^{23,24}$ In contrast, predominately horizontal programs are those which seek to integrate a particular health care technology into local infrastructures and labor forces. To ensure that technology-driven improvements toward health outcomes are sustainable in resource-poor nations, each initiative should aim to start as, or transition into, an appropriately balanced horizontally initiative. The optimal balance between vertical and horizontal components has been termed a 'synergistic' approach.

\section{A Synergistic Approach to Al based global health initiatives}

We propose a conceptual framework to guide sustainable Al-driven GHI. Figure I provides a breakdown of four key components for the sustainable development of an $\mathrm{Al}$-driven $\mathrm{GHI}$ : 
(I) Intervention, (2) Al Application, (3) Resource Sustainability Considerations, and (4) Synergistic Program Design. Intervention considerations include the type of healthcare problem one is seeking to solve. After establishing a type of intervention, the appropriateness of individual Al model types may be considered and targeted to optimize model fit. In Figure I, we chose well-regarded papers per intervention category (intelligent electronic records, ${ }^{10}$ biosurveillance, ${ }^{8,9,11-14,15,16,25}$ diagnostics, ${ }^{26-30}$ clinical decision assistance, ${ }^{7,19,20,31-35}$ and automated planning and scheduling, ${ }^{36}$ ) and investigated each paper to determine which Al application was chosen to meet the intervention's goal. After an Al model is selected, sustainability considerations must be made. For example, certain models may require more oversight or routine maintenance. Successful synergistic programs are ultimately founded upon a resource-conscious strategy which enables the transition from a predominately vertical program to one with optimal horizontal components.

Our second diagram (Figure 2) serves as a guide for developing a pre-implementation strategy for synergistic Al-driven GHI. Initial determination of which $\mathrm{Al}$ application is appropriate for a particular $\mathrm{GHI}$ helps to ensure that resource needs specific to that application are accounted for pre-deployment. We break down such resource considerations into the four general categories: (I) Model, (2) Personnel, (3) Infrastructure, and (4) Process.

We recognize that there is not a universal approach to Al implementation in resource poor regions. Our primary aim for this conceptual framework

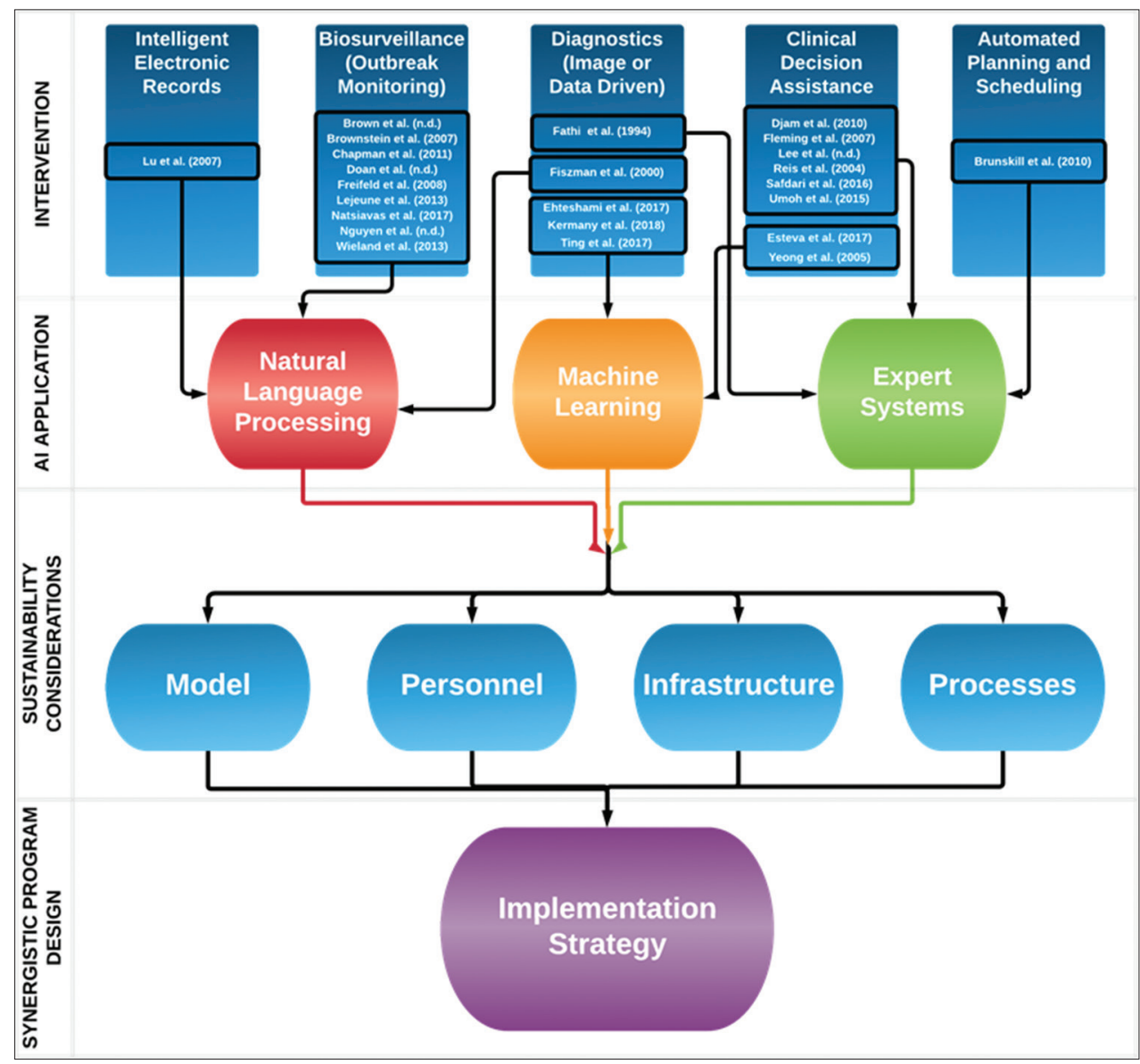

Figure I: A process for development of an artificial intelligence driven global health initiative. 


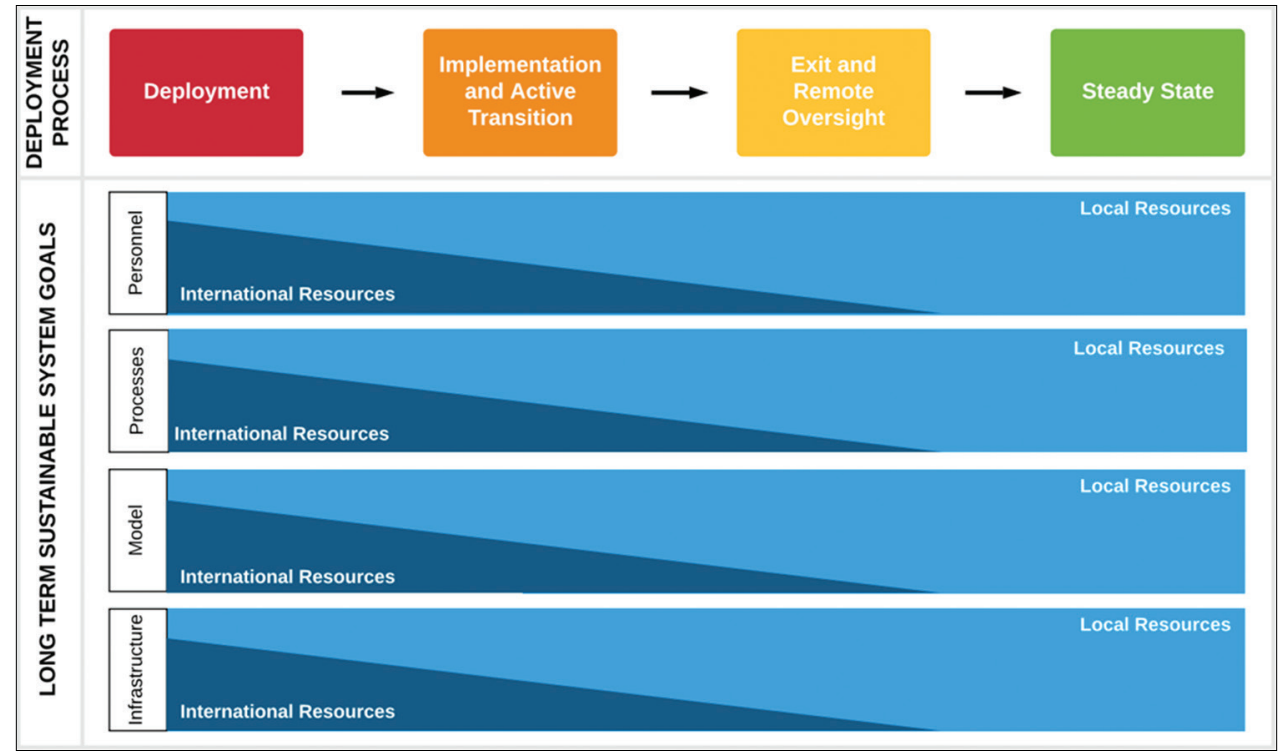

Figure 2: A potential strategy for implementation of a synergistic artificial intelligence driven global health initiative.

is to facilitate future research on sustainable Al-driven GHI. For example, one might start with a particular healthcare problem (within a resourcepoor setting) amenable to an Al-driven solution, select the appropriate $\mathrm{Al}$ application, consider that application's specific resource requirements, and then develop a sustainable strategy for implementation.

\section{Synergistic Strategy for Sustainability}

Al-based GHI must maintain focus on the end goal of establishing a self-sustaining model in their location of deployment. Therefore, prior to deployment, an ultimate exit strategy for the program must be well developed.At the time of deployment, it is expected that a large portion of the Al system, specifically the model, processes, personnel and infrastructure, may be provided primarily through international support. However, shortly after the program establishes itself in the region of interest, action should begin quickly to strengthen the local correlates of these key resources.

As the use of a particular Al application becomes fully operational, serious consideration should be given towards transitioning most, if not all, personnel and processes over to on-site assets in the region of interest. Appropriate preparations for personnel exchange should be planned at the time of initial deployment, as the transition will require training of local healthcare providers. Infrastructure limitations and technical model features will likely prevent expedient transition toward an optimal utilization of local resources. In some regions, transition to full reliance on a particular local resource could remain a challenge indefinitely. Even in such cases, a locally supported Al system should remain the goal. Achievement of steady-state for a program can be claimed when local infrastructure resources are able to supply the personnel needed to maintain the model and necessary processes with minimal reliance on outside support - the endpoint necessary to enact durable change.

\section{Discussion}

Overall, the available studies on Al-driven $\mathrm{GHI}$ are still extremely limited. Current literature is largely conceptual in nature, with a majority of studies outlining potential use cases for Al within particular resource-poor settings. However, as the application of medical Al reaches scale in these developed countries, it is critical for research on the sustainable implementation of this technology in resource-poor settings to grow in parallel. 
Our proposed synergistic model for Al-driven $\mathrm{GHI}$ used the four components distilled from numerous $\mathrm{GHI}-$ model, personnel, infrastructure, and processes - as inputs in a vertical-to-horizontal transition framework. The ultimate aim of this model is to enable any Al-driven $\mathrm{GHI}$ to seamlessly transition from a non-sustainable, internationaldependent intervention into a sustainable, locallydriven intervention capable of affecting permanent change over a large period of time.

We recognize the limitations inherent in the current form of this model. Further research and iterative refinements are necessary for this model to reach operational utility. A significant amount of data is still needed in order to conduct a proper analysis of the optimal time-to-transition from a vertical heavy program to a supportive horizontal program for each Al-driven GHI. We assert that these limitations may be addressed through the work of future studies who use our conceptual framework to further refine our model and define optimal transition points.

Future research in this area should begin by cataloging current $\mathrm{GHI}$ capable of being augmented by Al.To better understand the vertical to horizontal transition, we recommend a comprehensive analysis of the long-term resource and infrastructure demands for each Al application being considered for use in a GHI. Finally, we propose research to develop healthcare focused $\mathrm{Al}$ education programs to train local healthcare providers who would be involved in an Al-driven GHI.

\section{Conclusion and Global Health Implications}

Our model for the implementation of sustainable Al-driven $\mathrm{GHI}$ aims to provide a clear path toward

\section{Key Messages}

- Strategies for sustainable implementation artificial intelligence driven global health initiatives should be developed prior to its wide scale utilization.

- Future research on artificial intelligence driven global health initiatives should seek to characterize long-term resource and infrastructure needs. improved health outcomes in resource poor settings through the support of these developing countries as a whole. The potential for Al to transform the practice of medicine is now abundantly clear. Yet, amongst this excitement, we must not lose sight of our ultimate goal of empowering these developing nations to fully support themselves. In this new frontier of medicine, we stand to learn as much from the countries we help as they might from us.

\section{Compliance with ethical standards}

Conflicts of Interest: No Conflicts of Interest; Financial Disclosure: No disclosures; Funding/Support: No Funding; Ethics Approval: Not Applicable; Acknowledgements: None.

\section{References}

I. Topol EJ.High-performance medicine:the convergence of human and artificial intelligence. Nature Medicine. 2019;25(I):44-56. doi:I0.1038/s4I59|-0I8-0300-7.

2. Chan HP, Doi K, Vyborny CJ, Lam KL, Schmidt RA. Computer-aided detection of microcalcifications in mammograms. Methodology and preliminary clinical study. Investigational Radiology. 1988;23(9):664-67I.

3. Wang X, Peng Y, Lu L, Lu Z, Bagheri M, Summers RM. ChestX-Ray8: Hospital-Scale Chest X-Ray Database and Benchmarks on Weakly-Supervised Classification and Localization of Common Thorax Diseases. In: 2017 IEEE Conference on Computer Vision and Pattern Recognition (CVPR). Vol 20I7-Janua. IEEE; 2017:3462347I. doi:10.1 I09/CVPR.2017.369.

4. Nam JG, Park S, Hwang EJ, et al. Development and Validation of Deep Learning-based Automatic Detection Algorithm for Malignant Pulmonary Nodules on Chest Radiographs. Radiology.20 19;290(I):2 18-228. doi:I0.I |48/ radiol.2018180237.

5. Strodthoff N,StrodthoffC.Detecting and interpreting myocardial infarction using fully convolutional neural networks. Physiological Measurements. 2019; 40(I):0I500I. doi:10.1088/I36I-6579/aaf34d.

6. Wahl B, Cossy-Gantner A, Germann S, Schwalbe NR. Artificial intelligence (Al) and global health: how can Al contribute to health in resource-poor settings? BMJ Global Health. 2018;3(4):e000798. doi:10.1 I36/ bmigh-2018-000798.

7. Fleming G, Merwe M van der, McFerren G. Fuzzy expert systems and GIS for cholera health risk prediction in southern Africa. Environmental Model 
Software. 2007;22(4):442-448. doi:10.1016/J. ENVSOFT.2005.I2.008.

8. Natsiavas P., Maglaveras N., Koutkias V. (2017) A Public Health Surveillance Platform Exploiting FreeText Sources via Natural Language Processing and Linked Data: Application in Adverse Drug Reaction Signal Detection Using PubMed and Twitter. In: Riaño D., Lenz R., Reichert M. (eds) Knowledge Representation for Health Care. ProHealth 2016, KR4HC 2016. Lecture Notes in Computer Science, vol 10096. Springer, Cham.

9. Wieland ML,Wu ST,KaggalVC,Yawn BP.Tracking health disparities through natural-language processing. American Journal of Public Health. 2013;103(3):448449. doi: I0.2105/AJPH.20I2.300943.

10. Lu HM, King CC, Wu TS, et al. Chinese Chief Complaint Classification for Syndromic Surveillance. In: Intelligence and Security Informatics: Biosurveillance. Berlin, Heidelberg: Springer Berlin Heidelberg; 2007:I I-22. doi:10.1007/978-3-540-72608-I_2.

II. Lejeune G., Brixtel R., Lecluze C., DoucetA., Lucas N. (20I3) Added-Value of Automatic Multilingual Text Analysis for Epidemic Surveillance. In: Peek N., Marín Morales R., Peleg M. (eds.) Artificial Intelligence in Medicine. AIME 2013. Lecture Notes in Computer Science, vol 7885. Springer, Berlin, Heidelberg.

12. Doan S, Ngo QH, Kawazoe A, Collier N. Global Health Monitor-A Web-Based System for Detecting and Mapping Infectious Diseases. Proceedings of the Third International Joint Conference on Natural Language Processing:Volume-II, https://www.aclweb. org/anthology/108-2I40 Accessed April 20, 2019.

13. Chapman W.W., Gundlapalli A.V., South B.R., Dowling J.N. (20II) Natural Language Processing for Biosurveillance. In: Castillo-Chavez C., Chen H., Lober W., Thurmond M., Zeng D. (eds.) Infectious Disease Informatics and Biosurveillance. Integrated Series in Information Systems, vol 27. Springer, Boston, MA.

14. Brownstein J S, Freifeld C C. HealthMap: the development of automated real-time internet surveillance for epidemic intelligence. EuroSurveillance. 2007; I2(48):pii=3322. https://doi.org//0.2807/ esw. 1 2.48.03322-en.

15. Freifeld CC, Mandl KD, Reis BY, Brownstein JS. HealthMap: Global Infectious Disease Monitoring through Automated Classification and Visualization of Internet Media Reports. Journal of American Medical Informatics Association. 2008;15(2):150-157. doi:10.1 197/jamia.M2544.

16. Nguyen MT, Nguyen TT. Extraction of Disease Events for a Real-time Monitoring System. International Journal of ComputationalVision and Robotics, 2015;5(3) doi: I 0.I | 45/2542050.2542084.

17. Kiranantawat K, Sitpahul N, Taeprasartsit P, et al. The First Smartphone Application for Microsurgery Monitoring. Plastic Reconstruction Surgery. 20 I4; I 34(I): 130-139. doi:I0.1097/PRS.0000000000000276.

18. Gulshan V, Peng L, Coram M, et al. Development and Validation of a Deep Learning Algorithm for Detection of Diabetic Retinopathy in Retinal Fundus Photographs.Journal of theAmerican MedicalAssociation. 2016;316(22):2402. doi:10.100I/jama.2016.17216.

19. EstevaA,KuprelB,Novoa RA,etal.Dermatologist-level classification of skin cancer with deep neural networks. Nature. 2017;542(7639):II5-II8. doi:10.1038/ nature21056.

20. Yeong EK,HsiaoTC, Chiang HK, Lin C-W.Prediction of burn healing time using artificial neural networks and reflectance spectrometer. Burns. 2005;3 I (4):4I 5- 420. doi:I0.1016/J.BURNS.2004.12.003.

21. Onu CC, Udeogu I, Ndiomu E, et al. Ubenwa: Crybased Diagnosis of Birth Asphyxia. November 2017. http://arxiv.org/abs/1711.06405. Accessed May 29, 2019.

22. Jawhari B, Ludwick D, Keenan L, Zakus D, Hayward R. Benefits and challenges of EMR implementations in low resource settings: a state-of-the-art review. BMC Medical Informatics Decision Making. 2016;16(I): I16. doi:I0.1 |86/s I29||-016-0354-8.

23. Msuya J. Horizontal and Vertical Delivery of Health Services: What Are The Trade Offs?; 2694. http://documents.worldbank.org/curated/ en/9|449|46876/944686/Horizontal-and-verticaldelivery-of-health-services-what-are-the-trade-offs.

24. Atun RA, Bennett S, Duran A. When Do Vertical (Stand-Alone) Programmes Have a Place in Health Systems?; 2008. http://www.euro.who.int/pubrequest. Accessed June 9, 2019.

25. Brown P, Oktay C, Cevik A, et al. Sensitivity and Specificity of an Ngram Method for Classifying Emergency Department Visits into the Respiratory Syndrome in theTurkish Language.Academic Emergency Medicine, 2007; 10.1 197/j.aem.2007.03.1230.

26. Fathi-Torbaghan M, Meyer D. MEDUSA: A Fuzzy Expert System for Medical Diagnosis of Acute 
Abdominal Pain. Methods of Information in Medicine. 1994;33(5):522-529. doi:10.1055/s-0038-1635055.

27. Fiszman M, Chapman WW,Aronsky D, Evans RS, Haug PJ.Automatic Detection of Acute Bacterial Pneumonia from Chest X-ray Reports. Journal of American Medical Informatics Association. 2000;7(6):593-604. doi: I0.1 I 36/ jamia.2000.0070593.

28. Ehteshami Bejnordi B, Veta $M$, Johannes van Diest $\mathrm{P}$, et al. Diagnostic Assessment of Deep Learning Algorithms for Detection of Lymph Node Metastases in Women With Breast Cancer. Journal of the American Medical Association. 2017;318(22):2199. doi:I0.100I/jama.2017.I4585.

29. Kermany DS, Goldbaum M, Cai W, et al. Identifying Medical Diagnoses and Treatable Diseases by ImageBased Deep Learning. Cell. 2018;172(5): I |22-II3I. e9. doi:I0.1016/J.CELL.2018.02.010.

30. Ting DSW, Cheung CY-L, Lim G, et al. Development and Validation of a Deep Learning System for Diabetic Retinopathy and Related Eye Diseases Using Retinal Images From Multiethnic Populations With Diabetes. Journal of the American Medical Association. 2017;318(22):221 I. doi:10.1001/jama.2017.18I52.

31. Djam X,Wajiga G, Kimbi Y, Blamah N. A Fuzzy Expert
System for the Management of Malaria. International Journal of Pure and Applied Sciences and Technology. 20I I; 5(2), 84- 108.

32. Lee CS,Wang MHA Fuzzy Expert System for Diabetes Decision Support Application, IEEE Transactions on Systems, Man, and Cybernetics, Part B (Cybernetics). 4I (I). I39-|53.

33. Reis MAM, Ortega NRS, Silveira PSP. Fuzzy expert system in the prediction of neonatal resuscitation. Brazilian Journal of Medical and Biological Research = Rev Bras Pesqui medicas e Biol. 2004;37(5):755-764.

34. Safdari R, Kadivar M, Langarizadeh $M$, Nejad AF, Kermani F. Developing a Fuzzy Expert System to Predict the Risk of Neonatal Death. Acta Inform Med. 20I6;24(I):34-37. doi:I0.5455/aim.20I6.24.34-37.

35. Umoh UA, Umoh U, Nyoho E. A Fuzzy Intelligent Framework for Healthcare Diagnosis and Monitoring of Pregnancy Risk Factor in Women. Journal of Health, Medicine and Nursing. 2015; 18, 2015.

36. Brunskill E, Lesh N. Routing for Rural Health: Optimizing Community HealthWorkerVisit Schedules. 2010; AAAI Spring Symposium - Technical Report. https:/www.aaai.org/ocs/index.php/SSS/SSSI0/paper/ viewPaper/II 39.Accessed June 3, 2019. 